\title{
Comparison of chromosome analysis and chromosomal microarray analysis: what is the value of chromosome analysis in today's genomic array era?
}

\author{
Weimin Bi, PhD'1, Caroline Borgan, BS 1 , Amber N. Pursley, MS¹, Patricia Hixson, PhD¹, \\ Chad A. Shaw, PhD1', Carlos A. Bacino, MD 1,2, Seema R. Lalani, MD ${ }^{1,2}$, Ankita Patel, PhD ${ }^{1}$, \\ Pawel Stankiewicz, MD, PhD ${ }^{1}$, James R. Lupski, MD, PhD ${ }^{1-3}$, Arthur L. Beaudet, $\mathrm{MD}^{1-3}$ \\ and Sau Wai Cheung, PhD, MBA ${ }^{1}$
}

Purpose: Chromosomal microarray analysis enables the detection of microdeletions/duplications and has become the standard in clinical diagnostic testing for individuals with congenital anomalies and developmental disabilities. In the era of genomic arrays, the value of traditional chromosome analysis needs to be reassessed.

Methods: We studied 3,710 unrelated patients by chromosomal microarray analysis and chromosome analysis simultaneously and compared the results.

Results: We found that chromosomal microarray analysis detected the chromosomal imbalances that were identified by chromosome analysis with the exception of six cases $(0.16 \%)$ that had mosaic abnormalities. Of note, one case showed mosaicism for two abnormal cell lines, resulting in a balanced net effect and a normal chromo- somal microarray analysis. Further structural abnormalities such as unbalanced translocations, rings, and complex rearrangements were subsequently clarified by chromosome analysis in $18 \%$ of the cases with abnormal chromosomal microarray analysis results. Apparently balanced rearrangements were detected by chromosome analysis in 30 cases $(0.8 \%)$.

Conclusion: Our data demonstrate that although chromosomal microarray analysis should be the first-tier test for clinical diagnosis of chromosome abnormalities, chromosome analysis remains valuable in the detection of mosaicism and delineation of chromosomal structural rearrangements.

Genet Med 2013:15(6):450-457

Key Words: array CGH; chromosome analysis; chromosome structural rearrangement; developmental disability; FISH; mosaicism

\section{INTRODUCTION}

The development and implementation of genomic microarray technology have ushered in a new era in clinical genetics. Previously, G-banded chromosome analysis had been the standard for clinical diagnosis of chromosome abnormalities for more than 35 years. Chromosome analysis detects numerical and structural chromosomal abnormalities through counting chromosomes and analyzing chromosomal banding patterns in metaphase cells. To be detectable by chromosome analysis, the structural changes have to be $>3-10 \mathrm{Mb}$ in size. Fluorescence in situ hybridization (FISH) can also detect chromosome abnormalities, including those beyond the resolution of chromosome analysis, through examination of metaphase or interphase cells. A disadvantage of FISH is that it requires prior knowledge of the specific region that might be abnormal and therefore has limited utility as a first-tier test for clinical diagnosis. Chromosomal microarray analysis (CMA), one of the most frequently used microarray technologies in clinical laboratories, detects chromosomal losses and gains throughout the genome by comparing the hybridization intensities between a patient's
DNA and a normal control's DNA. FISH analysis, chromosome analysis, or molecular techniques are usually performed after CMA to confirm the results and identify translocations or insertions associated with the copy number changes. ${ }^{1,2}$

An advantage of CMA is that it enables the detection of losses and/or gains of chromosomal material that are submicroscopic, i.e., too small to be detectable by conventional G-banding chromosome analysis, which leads to a significantly increased diagnostic yield of $\sim 10 \%$ higher for individuals with unexplained developmental disabilities or congenital anomalies. ${ }^{3,4}$ In addition, CMA analyzes DNA extracted from uncultured cells of all different types, which has fewer experimental requirements for sample quality and usually shortens the reporting time for results as compared with chromosome analysis. Although detection of somatic mosaicism by CMA that was missed by chromosome analysis has been reported, ${ }^{5,6}$ mosaicism $<30 \%$ cannot be reliably detected by CMA. In addition, CMA cannot detect apparently balanced rearrangements.

A consensus statement released by the American College of Medical Genetics and Genomics recommended chromosomal 
microarray as a first-tier clinical diagnostic test for congenital developmental disorders, ${ }^{4}$ making it debatable whether traditional chromosome analysis is still necessary. ${ }^{7}$ Until now, no large-scale studies have been performed to investigate chromosome abnormalities that are detectable by chromosome analysis but undetectable or likely to be missed by CMA. In this study, we identified 3,710 postnatal cases in which both chromosome and chromosomal microarray analyses were

Table 1 Summary of the results of chromosome analysis and CMA

\begin{tabular}{|lcc|} 
Chromosome analysis & $\begin{array}{c}\text { Number of } \\
\text { cases }\end{array}$ & $\begin{array}{c}\text { Percentage of } \\
\text { all cases (\%) }\end{array}$ \\
\hline Normal & 3,385 & 91.2 \\
\hline Abnormal CMA & 179 & 4.8 \\
\hline Normal CMA & 3,206 & 86.4 \\
\hline Abnormal & 325 & 8.8 \\
\hline Unbalanced (net loss and/or gain) & 295 & 8.0 \\
\hline Abnormal CMA & 289 & 7.8 \\
\hline Normal CMA & 6 & 0.16 \\
\hline Balanced (structural abnormalities) & 30 & 0.8 \\
\hline Abnormal CMA & 1 & 0.03 \\
\hline Normal CMA & 29 & 0.8 \\
\hline Total cases & 3,710 & 100 \\
\hline
\end{tabular}

CMA, chromosomal microarray analysis. performed simultaneously in a clinical setting. The results show that CMA detected the unbalanced chromosome abnormalities that were identified by chromosome analysis with the exception of six cases $(0.16 \%)$ due to low-level mosaicism or no net genomic imbalance. In addition, chromosome analysis provided valuable information about structural rearrangements not readily delineated by CMA.

\section{Patients}

\section{MATERIALS AND METHODS}

From 2004 to 2011, CMA was performed on genomic DNA isolated from the blood of $>40,000$ postnatal cases referred to the Medical Genetics Laboratories at the Baylor College of Medicine. Included in this study were the 3,710 consecutive cases which had chromosome analysis performed simultaneously with CMA.

\section{CMA}

Peripheral blood was collected in a purple-top tube containing EDTA as an anticoagulant. Genomic DNA was extracted from whole blood manually using the Puregene DNA Blood Kit (Gentra, Minneapolis, MN). CMA was performed according to the manufacturers' instructions, with minor modifications as described previously. ${ }^{8,9}$ The arrays used evolved from bacterial artificial clone arrays (V3-V6), which were targeted arrays with increased coverage in known disease regions but limited coverage in the backbone, to whole-genome $105 \mathrm{~K}$

Table 2 Cases with an abnormal chromosome analysis and a normal CMA at the time of diagnosis

\begin{tabular}{|c|c|c|c|c|c|c|}
\hline Case ID & $\begin{array}{l}\text { Karyotyping } \\
\text { findings }\end{array}$ & G-banding results & $\begin{array}{l}\text { Mosaicism } \\
\text { level (\%) }\end{array}$ & Indication & $\begin{array}{c}\text { CMA } \\
\text { version }\end{array}$ & Notes \\
\hline 1 & Aneuploidy & $47, X X,+21[4] / 46, X X[49]$ & 7.5 & DF, abnormal gait & $V 5^{\mathrm{a}}$ & \\
\hline 2 & Aneuploidy & $47, X X,+9[4] / 45, X[2] / 47, X X X[1] / 46, X X[93]$ & 4.0 & $\begin{array}{l}\text { Failure to thrive, } \\
\text { speech delay }\end{array}$ & $V 5^{a}$ & $\begin{array}{l}\text { Previous karyotyping: } \\
45, X[2] / 46, X X[98]\end{array}$ \\
\hline 3 & Aneuploidy & $47, X X X[2] / 48, X X X X[1] / 45, X[1] / 46, X X[96]$ & 2.0 & MCA & $\vee 7^{b}$ & \\
\hline 4 & $\begin{array}{l}\text { Marker } \\
\text { chromosome }\end{array}$ & $47, \mathrm{XY},+\operatorname{mar}[4] / 46, \mathrm{XY}[59]$ & 6.3 & $\begin{array}{l}\text { Mild DD, possible Fabry } \\
\text { disease }\end{array}$ & $V 8^{b}$ & \\
\hline 6 & $\begin{array}{l}\text { Two abnormal } \\
\text { cell lines }\end{array}$ & $\begin{array}{l}45, X[14] / 46, X, \operatorname{idic}(Y)(q 12) . i s h \operatorname{idic}(Y) \\
(S R Y++, D Y Z 1-)[7]\end{array}$ & 66.7 & $\begin{array}{l}\text { Unspecified congenital } \\
\text { anomaly of genitalia }\end{array}$ & $V 8^{b}$ & $\begin{array}{l}\text { FISH showed an ad- } \\
\text { ditional cell line with } \\
\text { a normal } Y \text { and an } \\
\text { idic(Y) in } 6 \% \text { cells }\end{array}$ \\
\hline 9 & $\begin{array}{l}\text { Complex } \\
\text { rearrangement }\end{array}$ & $\begin{array}{l}46, X Y, \operatorname{der}(2) \operatorname{inv}(2)(p 12 q 31.1) \operatorname{ins}(2 ; 17) \\
(q 31.1 ; q 22 q 24)\end{array}$ & NA & $\mathrm{DD} / \mathrm{MR}$, short stature & $V 5^{a}$ & $\begin{array}{l}\text { Deletion in } 2 \mathrm{q} 31.1 \\
\text { detected by } \mathrm{V} 8 \mathrm{CMA}\end{array}$ \\
\hline 10 & $\begin{array}{l}\text { Complex } \\
\text { rearrangement }\end{array}$ & $\begin{array}{l}46, X Y, \operatorname{der}(6) \operatorname{ins}(6 ; 2)(p 23 ; q 24.1 q 14.2) \operatorname{del}(6) \\
(q 16.3 q 21), t(12 ; 18)(p 12.2 ; q 12.2) d n\end{array}$ & NA & MCA, DF & $V 5^{a}$ & $\begin{array}{l}\text { Deletions detected } \\
\text { by V8 CMA }\end{array}$ \\
\hline
\end{tabular}

No copy number changes were detected in cases 7-10 using a targeted array. However, the abnormalities were detected using the current wholegenome array (V8 CMA).

CMA, chromosomal microarray analysis; DD, developmental delay; DF, dysmorphic features; FMHX of chrom abn, family history of chromosome abnormality; MCA, multiple congenital anomalies; MR, mental retardation; NA, not applicable.

a Targeted array. ${ }^{b}$ Whole-genome array. 
oligonucleotide arrays $(\mathrm{V} 7)^{10}$ and the current version of the whole-genome array (V8), which has $\sim 180,000$ oligonucleotides including exon coverage for 1,700 disease or candidate genes. ${ }^{11}$ None of the arrays used were intended to detect copy number-neutral regions of absence of heterozygosity. FISH analysis was performed to confirm the CMA results as described previously. ${ }^{3}$ For a copy number loss, five metaphase cells were examined. For a copy number gain, 50 nuclei were examined independently by two technologists in addition to an examination of at least one metaphase cell to rule out a translocation or an insertion of the gained material.

\section{Chromosome analysis}

Chromosome analysis was performed using standard protocols for the Giemsa (GTG) banding technique. Briefly, cells were treated with phytohemagglutinin to stimulate the growth of $\mathrm{T}$ lymphocytes. When a chromosome abnormality was identified in a single cell out of the 20 cells examined, additional cells up to 100 were analyzed to rule out low-level mosaicism.

\section{RESULTS}

The results of G-banded chromosome analysis of the 3,710 unrelated cases are summarized in Table 1. A normal chromosome constitution was identified in 3,385 (91.2\%) patients, of which $179(4.8 \%, 179 / 3,710)$ patients had abnormalities detected by CMA.

Chromosome analysis detected unbalanced abnormalities in 295 cases (8\%). CMA results were normal at the time of diagnosis for 10 cases with abnormalities identified by chromosome analysis (Table 2) whereas they were consistent with the chromosome analysis findings in the remaining 285 cases $(96.6 \%, 285 / 295)$. CMA was repeated using a whole-genome array (V8) in 4 of the 10 cases (cases 7-10 in Table 2; Figure 1) and copy number changes relevant to the chromosome findings were detected. Thus, only six of the 3,710 cases $(0.16 \%)$ studied had unbalanced abnormalities detected by chromosome analysis but not found by CMA. Chromosome analysis identified simple balanced translocations or inversions in 30 cases $(0.8 \%)$, leading to an overall

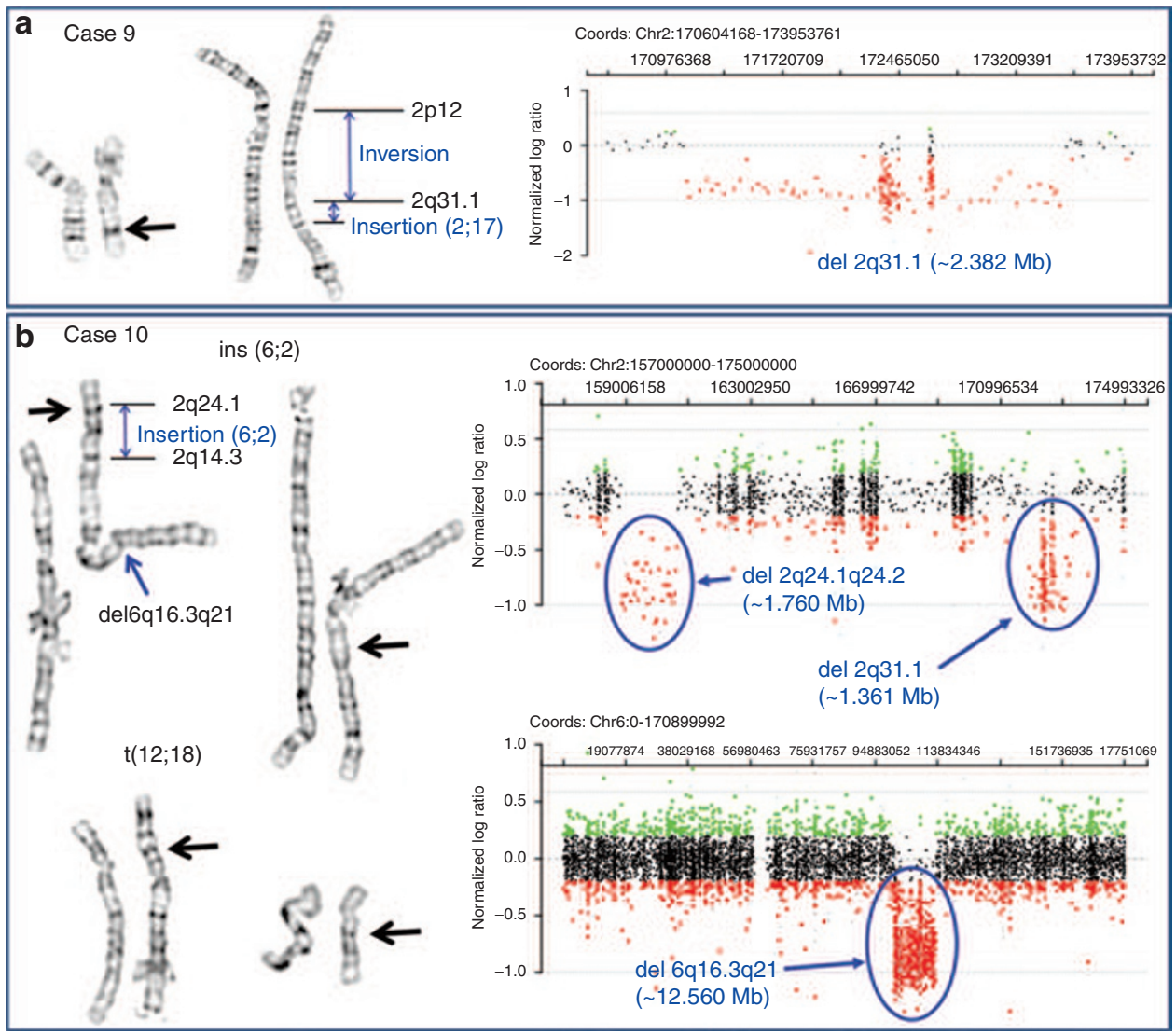

Figure 1 Complex rearrangements characterized by a combination of chromosome analysis and chromosomal microarray analysis (CMA). (a) In case 9 , a deletion in the long arm of one chromosome 17 was detected by G-banded chromosome analysis. CMA revealed no copy number change for chromosome 17 but a copy number loss of $\sim 2.382 \mathrm{Mb}$ in chromosome 2 at band 2q31.1. Retrospective examination of the chromosome analysis showed an insertion (ins) of the 17q genomic material into the short arm of one chromosome 2. In addition, in the same chromosome 2, there is a pericentric inversion between $2 \mathrm{p} 12$ and 2q31.1. The deletion detected by CMA is at or near the inversion breakpoint. (b) In case 10, an insertion of a portion of the long arm of one chromosome 2 into the short arm of one chromosome 6 was detected by G-banded chromosome analysis. In addition, there is an apparently balanced reciprocal translocation between chromosomes 12 and 18. CMA revealed two copy number losses on chromosome 2 separated by an $\sim 11.3$ Mb copy-neutral segment. The copy number loss on 2q24 is located at or near one insertion breakpoint. In addition, CMA detected a copy number loss of $~ 12.560 \mathrm{Mb}$ in the long arm of chromosome 6 . No copy number changes were detected in chromosomes 12 or 18 in this version 8 array. The abnormal chromosomes are indicated by arrows. 
detection rate for chromosome analysis of $8.8 \%(325 / 3,710)$. None of the balanced cases had copy number changes at or near the breakpoints detected by clinical CMA using either targeted or whole-genome arrays. One case had an abnormal gain not detectable by chromosome analysis on a chromosome apparently not involved in the translocation.

The overall detection rate for CMA using both targeted arrays (V4-V6) and whole-genome arrays (V7 and V8) in this cohort was $12.6 \%$, which is significantly higher than that of chromosome analysis $(8.8 \%)\left(P\right.$ value $<0.0001$ by $\chi^{2}$ analysis). The CMA detection rate for the whole-genome array was $14.5 \%$ as compared with $10.9 \%$ for the targeted array.

\section{Mosaic imbalances detected by chromosome analysis but missed by CMA}

All six of the cases with unbalanced chromosome abnormalities detected by chromosome analysis but not by CMA showed mosaicism. The level of mosaicism for cases 1-4 was very low, ranging between $2 \%$ and $7.5 \%$ (Table 2), which is below the detection limit of CMA. The low level of mosaicism in these cases cannot be reliably detected by a single-nucleotide polymorphism array either. ${ }^{12}$ Case 5 showed mosaicism for a derivative chromosome 8 , resulting from an unbalanced translocation between the long arms of one chromosome 8 and one chromosome 21 in $30 \%$ of the cultured blood cells. The derivative chromosome 8 resulted in partial trisomy for the distal portion of chromosome 21, which was not detected by CMA. Case 6 had two abnormal cell lines including a 45 ,X cell line in $67 \%$ of cells and another cell line having one chromosome $\mathrm{X}$ and one isodicentric $\mathrm{Y}$ chromosome with break and fusion points on band Yq12 in the remaining 33\% of cells. The 45,X cell line led to a loss of chromosome Y, whereas the isodicentric Y cell line led to a gain of chromosome $\mathrm{Y}$, except for the heterochromatic and pseudoautosomal region in the long arm, resulting in a balanced net effect and an apparently normal result on CMA.

\section{Detection of mosaicism can be maximized by using both CMA and chromosome analysis}

Mosaic abnormalities were detected by chromosome analysis and/or CMA in $43(1.2 \%)$ cases. Mosaicism was detected by both chromosome analysis and CMA in half of these cases (49\%, 21/43). Mosaicism was detected by chromosome analysis only, through examination of 20-100 cells, in 17 cases (39\%; 17/43), six of them had a normal CMA (cases 1-6 in Table 2). The remaining 11 cases had an abnormal CMA, but the log ratio plot did not suggest mosaicism because the level of mosaicism for the abnormal cell line was very high ( $\geq 80 \%)$, as exemplified in one mosaic trisomy 18 case (Figure 2a,b).

CMA detected mosaicism for autosomal chromosomes in which chromosome analysis was found to be normal in five cases $(12 \% ; 5 / 43)$. Two of these cases were reported previously as case 8 (mosaic trisomy 14) and 9 (mosaic trisomy 22). ${ }^{6}$ Mosaicism was suspected by the CMA plot, with log ratios ranging between 0.04 and 0.22 and confirmed by FISH analysis on a blood smear or phytohemagglutinin-stimulated T cells showing mosaicism levels ranging from $6.5 \%$ to $29 \%$. The log ratios correlate with the percentage of trisomic cells revealed by FISH, except for case $9,{ }^{6}$ who had a lower level of trisomic cells by FISH than expected by the log ratio, probably because the FISH analysis was performed on a 2-week-old uncultured blood sample. Further analysis of a fresh sample was not available as the infant passed away shortly after birth. Of note, one case showed trisomy 9 in $29 \%$ of cells in an unstimulated blood sample by FISH analysis whereas no trisomy 9 cells were observed in the 50 metaphase cells examined from stimulated T-cell cultures by chromosome analysis.

Chromosomal structural abnormalities detected by CMA are further characterized by chromosome analysis and/or FISH

Most of the copy number gains and losses detected by CMA are aneuploidy, simple deletions, or tandem duplications. However, some of these copy number changes are associated with additional chromosomal structural rearrangements that can only be defined by traditional cytogenetic analysis. Of the 469 cases with an abnormal CMA result, structural rearrangements in addition to simple deletions or duplications were observed under the microscope in 85 (18\%) cases (Table 3). Approximately half $(51 \% ; 44 / 85)$ of these cases involved an unbalanced translocation or insertion whereas one-third $(33 \% ; 28 / 85)$ had a marker or ring chromosome, or isochromosome or isodicentric chromosome. The remaining $16 \%(13 / 85)$ of the cases had a complex rearrangement. FISH was performed in 38 of these 85 cases, which revealed chromosomal structural abnormalities consistent with the chromosome analysis. The structural rearrangements observed in the remaining cases are expected to be detectable by FISH, except for three of the complex rearrangements. The complex rearrangements not detectable by FISH include cases 9 and 10 in Table 2 and one case with a deletion at a translocation breakpoint. Without chromosome analysis, the complex rearrangements would be misinterpreted as simple deletions by both CMA and FISH.

As expected, most of the cases with two or more copy number changes, including $88 \%$ (23/26) of the cases involving one chromosome and $87 \%$ (33/38) of the cases involving two chromosomes, had chromosomal structural rearrangements visible under the microscope. For copy number changes involving two chromosomes, the vast majority of the rearrangements were unbalanced translocations between the two chromosomes resulting in a derivative chromosome. One patient with a complex rearrangement had two copy number changes that are apparently not related; CMA detected two interstitial losses, one on chromosome $1 \mathrm{p}$ and one on chromosome 10p (Figure 2e). Chromosome analysis revealed the loss on 10p was a simple deletion, whereas the loss on $1 \mathrm{p}$ was at the breakpoint of an apparently balanced translocation between chromosomes 1 and 3 .

Additional structural rearrangements were more likely to be observed in cases with a single copy number gain (17/91; 19\%) than in those with a single copy number loss $(8 / 214 ; 4 \%)$. Most of the rearrangements associated with a gain are unbalanced translocations and supernumerary marker chromosomes. In 

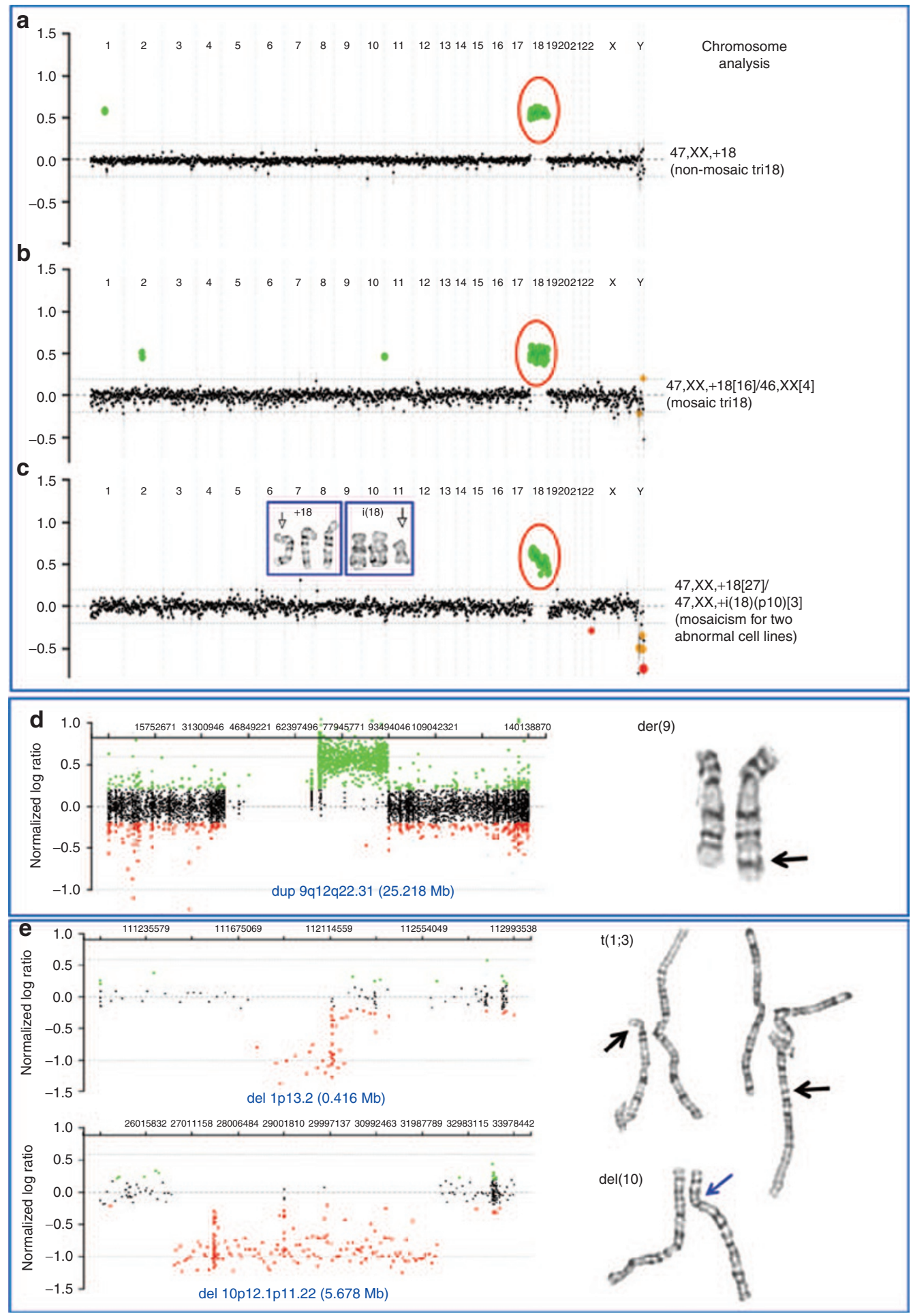

Figure 2 Chromosome analysis facilitated the detection of mosaicism and chromosomal structural rearrangements. (a-c) Chromosomal microarray analysis (CMA) showed a similar gain in copy number for all probes specific for chromosome 18 in three separate samples. However, chromosome analysis revealed a different finding for each case. (a) Trisomy 18 with no mosaicism detected by chromosome analysis. (b) Mosaicism with a trisomy 18 cell line in $80 \%$ of cells whereas the remaining $20 \%$ of cells are normal 46,XX. (c) Mosaicism for two abnormal cell lines. One cell line had trisomy 18 in $91 \%$ of cells and the other cell line had an isochromosome 18 consisting of two copies of the short arm of chromosome 18 in $9 \%$ of cells. (d,e) Examples of chromosomal structural rearrangements associated with interstitial copy number changes. (d) An interstitial copy number gain of $\sim 25.218 \mathrm{Mb}$ was detected by CMA. G-banded chromosome analysis showed that the duplicated segment involving chromosome bands 9q12q22.31 was inserted into the distal long arm of chromosome 9 at band 9q33. The abnormal chromosomes are indicated by arrows. (e) Two interstitial copy number losses were detected by CMA; one was $\sim 0.416 \mathrm{Mb}$ in chromosome $1 \mathrm{p}$ and the other was $\sim 5.678 \mathrm{Mb}$ in chromosome 10p. G-banded chromosome analysis showed that the copy number loss in $1 \mathrm{p}$ was adjacent to the breakpoint of a translocation between the short arm of one chromosome 1 and the long arm of one chromosome 3. In contrast, the deletion in 10p is a simple deletion. 
Table 3 Summary of structural rearrangements detected by chromosome analysis in the cases with an abnormal CMA

\begin{tabular}{|c|c|c|c|c|c|c|}
\hline \multirow[b]{2}{*}{ Abnormal CMA results } & \multirow[b]{2}{*}{$\begin{array}{c}\text { Number of } \\
\text { cases }\end{array}$} & \multicolumn{5}{|c|}{ Cases with additional structural rearrangements } \\
\hline & & $\begin{array}{l}\text { Translocation/ } \\
\text { insertion }\end{array}$ & $\begin{array}{l}\text { Marker/ring/ } \\
\text { isochromosome/ } \\
\text { isodicentric } \\
\text { chromosome }\end{array}$ & Complex & $\begin{array}{l}\text { Total } \\
\text { cases }\end{array}$ & $\%$ \\
\hline Entire chromosome & 100 & 1 & 3 & 0 & 4 & 4 \\
\hline Single segmental deletion & 214 & 3 & 4 & 1 & 8 & 4 \\
\hline Two or more CNVs in two chromosome & 38 & 27 & 5 & 1 & 33 & 87 \\
\hline Total cases & 469 & 44 & 28 & 13 & 85 & 18 \\
\hline
\end{tabular}

All the structural rearrangements are expected to be observable by FISH analysis except for three cases involving complex rearrangements.

CMA, chromosomal microarray analysis; CNV, copy number variant; FISH, fluorescence in situ hybridization.

addition, two cases had a single interstitial copy number gain, with the duplicated segment inserted into a different genomic location. One of these cases had a copy number gain of $>25 \mathrm{Mb}$ in 9q12q22.31 detected by CMA. Chromosome analysis showed that the duplicated segment was inserted into the distal long arm of chromosome 9 at band 9q33 (Figure 2d).

Copy number changes involving one entire chromosome are usually indicative of aneuploidy; however, chromosome analysis showed structural rearrangements in four cases. An example of one of these cases is shown in Figure 2c. Although CMA revealed a gain in copy number detected by all clones for chromosome 18 , suggesting trisomy 18 , chromosome analysis revealed mosaicism for a cell line with an additional chromosome 18 in $90 \%$ of cells whereas the remaining $10 \%$ of cells showed an additional isochromosome composed of the short arm of chromosome 18, resulting in tetrasomy for the 18p10-pter segment.

\section{DISCUSSION}

Chromosome analysis and CMA are clinically useful diagnostic tools for detecting chromosomal abnormalities throughout the human genome. Currently, CMA is recommended as the firsttier test for intellectual disability and congenital defects, replacing the previous role of chromosome analysis. In this study, we compared the results of chromosome analysis and CMA in 3,710 cases to determine the value of performing chromosome analysis.

\section{Maximum detection of mosaicism by a conjunction of CMA and traditional cytogenetic analysis}

This study showed that $1.2 \%(43 / 3,710)$ of patients had a mosaic finding. Mosaicism was observed only by chromosome analysis in $39 \%$ of cases because of either a very low $(<10 \%)$ or very high $(>80 \%)$ percentage of abnormal cells whereas $12 \%$ of cases were detected only by CMA. The remaining $49 \%$ of cases were detected by both CMA and chromosome analysis.

The detection of mosaicism by CMA and that by chromosome analysis differ because of the difference in technology and cell population analyzed. CMA analyzes DNA extracted from all of the nucleated cells in peripheral blood including multiple cell lineages. In contrast, chromosome analysis is performed primarily on $\mathrm{T}$ lymphocytes stimulated by phytohemagglutinin. Studies have demonstrated that CMA may be more sensitive when abnormal cells fail to respond to mitogens and/or abnormalities are rare or absent in $\mathrm{T}$ cells, such as in PallisterKillian syndrome., ${ }^{5,6}$ Five cases of mosaic trisomy, involving chromosomes $8,9,14$, and 22, were detected only by CMA.

Although CMA can easily detect mosaicism at levels of $30 \%$ or greater, it is limited in routinely detecting mosaicism at levels of $<10 \% .{ }^{6,13,14}$ Other array platforms, such as single-nucleotide polymorphism arrays may be better capable of detecting mosaicism by the information obtained from the B-allele frequencies. ${ }^{15,16}$ Chromosome analysis can detect low-level mosaicism through the individual examination of large numbers of cells. Standard chromosome studies of 20 cells will rule out $14 \%$ mosaicism at the $95 \%$ confidence level. More cells can be examined when one or two abnormal metaphase cells are identified. However, the analysis of many individual cells is time consuming and labor intensive. If mosaicism is suspected or confirmed, FISH can be utilized to examine hundreds of individual interphase cells to determine the level of mosaicism; FISH is relatively less time consuming than chromosome analysis.

The abnormalities missed by CMA, excluding the apparently balanced rearrangements, account for $<0.2 \%$ of total cases in this study (Table 2). Most cases were undetectable by CMA because the proportion of abnormal cells was below the detection limit of CMA $(<10 \%)$. The clinical significance is unclear for the very low level mosaicism in cases 2 and 3 , and the small marker chromosome that contains exclusively pericentromeric repeat sequences in case 4 . The remaining abnormalities missed by CMA are associated with phenotypic abnormalities. It is not clear why the abnormality seen in $30 \%$ of cultured cells in case 5 was missed by CMA, but it is presumably due to the abnormal cells being overrepresented specifically in the $\mathrm{T}$ cells. The presence of two different cell lines with genomic gains and losses involving the same region led to a net genomic balance for that region, thereby, eluding detection by CMA, as shown in case 6 .

Detection of mosaicism by CMA relies on the deviation from the expected log ratios for deletion or duplication without 
mosaicism. The suspected mosaicism has to be confirmed by chromosome analysis on cultured cells or FISH analysis preferably using blood smears. In 11 cases studied here, CMA was able to detect the chromosome abnormality but was unable to detect that the case was mosaic because of a low percentage of normal cells or the presence of an isodicentric chromosome leading to segmental tetrasomy. For example, two cases had a copy number gain detected by all the probes for chromosome 18 by CMA (Figure 2), suggesting trisomy 18. In actuality, chromosome analysis showed that one case had trisomy 18 in all the cells (Figure 2a), whereas the other case had mosaicism for trisomy 18 in $80 \%$ of cells (Figure $2 \mathrm{~b}$ ). In addition, when two or more abnormal cell lines involve the same region, as exemplified in the case in Figure 2c, chromosome and/or FISH analysis is essential for a correct interpretation of the chromosome abnormalities.

\section{Chromosomal structural information is provided by chromosome analysis but not apparent by CMA}

CMA provides very reliable information on whether copy number gains and losses are present but does not provide positional or orientation information. Our studies showed that structural abnormalities were seen in $18 \%$ of the abnormal CMA cases. Half of the structural rearrangements identified in this study are unbalanced translocations/insertions, one-third are other structural changes such as ring chromosomes, marker chromosomes, isochromosome and isodicentric chromosomes, and the remaining $15 \%$ are complex rearrangements. Given that most of these structural aberrations involve subtelomeric regions, terminal copy number changes have a higher likelihood of having further structural abnormalities. ${ }^{17}$ In general, both chromosome analysis and FISH analysis are able to identify translocations, insertions, isochromosomes, and markers, with different strengths for each method. Although FISH can detect changes too small to be detectable by chromosome analysis, analysis of chromosome banding patterns provides additional information on the involved regions. In the case of complex rearrangements, combined FISH and chromosome analysis may be necessary to completely determine the complex structural changes. With this exception, FISH analysis following an abnormal finding by CMA is typically adequate to provide information about the nature of the copy number change.

Some chromosomal rearrangements could be missed even after both CMA and FISH. For example, an interstitial deletion in $2 \mathrm{q}$ was detected by CMA in case 9 (Figure 1a). Without chromosome analysis, the rearrangement appears to be a simple single deletion even after confirmatory FISH analysis. However, examination of the karyotype showed an abnormal chromosome 2 with an insertion of a segment from 17q23.1q23.3 into band 2 p11.2. In addition, the chromosome 2 with the inserted $17 \mathrm{q}$ segment also has a pericentric inversion between $2 \mathrm{p} 12$ and 2q31.1. The deletion on 2q31.1 detected by CMA most likely occurred at or near the inversion breakpoint on the long arm of one chromosome 2. In summary, case 9 had a complex rearrangement including a deletion in $2 \mathrm{q}$ detectable by CMA but not visible by chromosome analysis, as well as an insertion and an inversion involving chromosomes 2 and 17, detectable by chromosome analysis but not by CMA. Similarly, case 10 had de novo rearrangements involving four chromosomes including an insertion of the 2q14.2q24.1 segment to the short arm of one chromosome 6, which also has a paracentric inversion, and a reciprocal translocation between chromosomes 12 and 18. Copy number losses were identified by CMA near the breakpoints in chromosomes 2 and 6 (Figure $\mathbf{1 b}$ ). A correct diagnosis requires the combination of CMA and chromosome analysis. ${ }^{18}$

Identification of chromosomal structural rearrangements is indispensable for genetic counseling of the family. The genomic imbalances detected by CMA could be due to an unbalanced segregation product of a balanced translocation or insertion in one parent and, therefore, warrants a strong recommendation for performing parental studies. In addition, the chromosomal structural information provides a guide for which parental studies should be recommended. For the rearrangements that are likely to be products of a balanced rearrangement, parental FISH or chromosome analysis should be performed instead of parental CMA.

\section{Apparently balanced rearrangements with a normal CMA study}

In this study, single apparently balanced translocations or inversions without other chromosome abnormalities were detected in 30 cases $(\sim 0.8 \%)$ by chromosome analysis. It has been reported that $\sim 40 \%$ of patients with multiple congenital anomalies/mental retardation and a de novo apparently balanced translocation have cryptic abnormalities near the breakpoints, or unrelated to the breakpoints, which can be easily detected by CMA..$^{19,20}$ However, CMA did not detect any copy number changes near the breakpoints in these 30 cases. Several factors may contribute to this observation. Most of the rearrangements were inherited based on the parental studies of a subset of the cases, meaning they are more likely to be "truly" balanced. In addition, Robertsonian translocations, which usually do not result in copy number changes of euchromatin, were included in this study but excluded in the published studies. Moreover, small cryptic deletions/duplications adjacent to the breakpoints may be missed because the arrays used for most of these cases were targeted and not of sufficient genomic resolution to detect an imbalance.

Although the majority of balanced rearrangements are benign, de novo rearrangements are associated with a higher disease risk due to a cryptic deletion or duplication, gene or enhancer disruption, position effects, or epigenetic effect. The risk of having a serious congenital anomaly for de novo reciprocal translocations and inversions is $6.7 \% .^{21}$ The risk is expected to be lower for those cases with a normal CMA using a whole-genome array. Therefore, the simple balanced rearrangements detected in this study are less likely to be the cause of the patients' phenotypes.

\section{Evidence-based strategy for efficient detection of chromosome abnormalities}

To determine if and when chromosome analysis should be performed for the clinical diagnosis of chromosomal abnormalities, 
we examined the results of cases studied simultaneously by both CMA and chromosome analysis. Chromosomal abnormalities detected by chromosome analysis but completely missed by CMA were observed in $\sim 1 \%$ of cases, including apparently balanced rearrangements in $0.8 \%$ cases and imbalances associated with mosaicism in $0.16 \%$ cases. In addition, chromosome analysis facilitated the detection of chromosomal structural rearrangements in $18 \%$ of the cases with abnormal CMA results.

Although CMA provides information about copy number variation and mosaicism, only chromosome analysis or FISH provides the chromosomal structural information associated with these copy number changes and identifies some cases of mosaicism not detected by CMA. Advantages of FISH over chromosome analysis is that small $(<3-10 \mathrm{Mb})$ copy number changes can be detected and a large number of nuclei can be quickly analyzed, which is especially useful for the evaluation of mosaicism. Therefore, after an abnormal CMA, FISH analysis can be applied first to detect structural rearrangements associated with copy number changes and confirm mosaicism and determine the percentage of abnormal cells. When insertions or translocations are detected by FISH, chromosome analysis may be indicated to determine the involved chromosomes. Instead of standard chromosome analysis, which studies 20 metaphase cells from two cultures, a small-scale chromosome analysis of five cells from one culture is sufficient for this purpose. This strategy would detect all structural changes except for a few rare complex rearrangements.

For cases with a normal result by CMA, a full chromosome analysis may be considered if the patient has multiple congenital anomalies, dysmorphic features, and/or mental retardation reminiscent of a chromosomal syndrome or clinical manifestations indicative of potential mosaicism such as pigmentary abnormalities that are randomly distributed or that follow the lines of Blashko and growth asymmetry in association with intellectual disability. On the basis of this study, $\sim 1 \%$ of cases would have informative chromosome results, but $<0.001$ of cases $(3 / 3,710)$ would have a clinically significant finding.

In summary, our study further confirms that almost all of the chromosomal abnormalities detectable by chromosome analysis are detected by CMA, supporting the recommendation that CMA be the first-tier test. However, traditional cytogenetic analysis remains useful for the detection of mosaicism and characterization of structural rearrangements.

\section{ACKNOWLEDGMENTS}

We thank the staff in the Medical Genetics Laboratories, Baylor College of Medicine, for their contributions.

\section{DISCLOSURE}

J.R.L. is a consultant for Athena Diagnostics and Ion Torrent Systems, has stock ownership in 23andMe, and is a coinventor on multiple United States and European patents for DNA diagnostics.
The Department of Molecular and Human Genetics of the Baylor College of Medicine derives revenue from clinical testing by highresolution human genome analyses.

\section{REFERENCES}

1. Kang SH, Shaw C, Ou Z, et al. Insertional translocation detected using FISH confirmation of array-comparative genomic hybridization $(\mathrm{aCGH})$ results. Am J Med Genet A 2010;152A:1111-1126.

2. Neill NJ, Ballif BC, Lamb AN, et al. Recurrence, submicroscopic complexity, and potential clinical relevance of copy gains detected by array CGH that are shown to be unbalanced insertions by FISH. Genome Res 2011;21:535-544.

3. LuX, Shaw CA, Patel A, et al. Clinical implementation of chromosomal microarray analysis: summary of 2513 postnatal cases. PLOS ONE 2007;2:e327.

4. Miller DT, Adam MP, Aradhya S, et al. Consensus statement: chromosomal microarray is a first-tier clinical diagnostic test for individuals with developmental disabilities or congenital anomalies. Am J Hum Genet 2010;86:749-764.

5. Ballif BC, Rorem EA, Sundin K, et al. Detection of low-level mosaicism by array CGH in routine diagnostic specimens. Am J Med Genet A 2006;140:27572767.

6. Cheung SW, Shaw CA, Scott DA, et al. Microarray-based CGH detects chromosomal mosaicism not revealed by conventional cytogenetics. Am J Med Genet A 2007;143A:1679-1686.

7. Bui TH, Vetro A, Zuffardi O, Shaffer LG. Current controversies in prenatal diagnosis 3: is conventional chromosome analysis necessary in the post-array CGH era? Prenat Diagn 2011;31:235-243.

8. Cheung SW, Shaw CA, Yu W, et al. Development and validation of a CGH microarray for clinical cytogenetic diagnosis. Genet Med 2005;7:422-432.

9. Ou Z, Kang SH, Shaw CA, et al. Bacterial artificial chromosome-emulation oligonucleotide arrays for targeted clinical array-comparative genomic hybridization analyses. Genet Med 2008;10:278-289.

10. El-Hattab AW, Smolarek TA, Walker ME, et al. Redefined genomic architecture in $15 q 24$ directed by patient deletion/duplication breakpoint mapping. Hum Genet 2009;126:589-602.

11. Boone PM, Bacino CA, Shaw CA, et al. Detection of clinically relevant exonic copy-number changes by array CGH. Hum Mutat 2010;31:1326-1342.

12. Bruno DL, White SM, Ganesamoorthy D, et al. Pathogenic aberrations revealed exclusively by single nucleotide polymorphism (SNP) genotyping data in 5000 samples tested by molecular karyotyping. J Med Genet 2011;48:831-839.

13. Hoang S, Ahn J, Mann K, et al. Detection of mosaicism for genome imbalance in a cohort of 3,042 clinical cases using an oligonucleotide array CGH platform. Eur J Med Genet 2011;54:121-129.

14. Neill NJ, Torchia BS, Bejjani BA, Shaffer LG, Ballif BC. Comparative analysis of copy number detection by whole-genome BAC and oligonucleotide array $\mathrm{CGH}$. Mol Cytogenet 2010;3:11

15. Conlin LK, Thiel BD, Bonnemann CG, et al. Mechanisms of mosaicism, chimerism and uniparental disomy identified by single nucleotide polymorphism array analysis. Hum Mol Genet 2010;19:1263-1275.

16. Papenhausen $P$, Schwartz $S$, Risheg $H$, et al. UPD detection using homozygosity profiling with a SNP genotyping microarray. Am J Med Genet A 2011;155A:757768.

17. Shao L, Shaw CA, Lu XY, et al. Identification of chromosome abnormalities in subtelomeric regions by microarray analysis: a study of 5,380 cases. Am J Med Genet A 2008;146A:2242-2251.

18. Liu P, Erez A, Nagamani SC, et al. Chromosome catastrophes involve replication mechanisms generating complex genomic rearrangements. Cell 2011;146:889-903.

19. De Gregori M, Ciccone R, Magini P, et al. Cryptic deletions are a common finding in "balanced" reciprocal and complex chromosome rearrangements: a study of 59 patients. J Med Genet 2007:44:750-762.

20. Schluth-Bolard C, Delobel B, Sanlaville D, et al. Cryptic genomic imbalances in de novo and inherited apparently balanced chromosomal rearrangements: array CGH study of 47 unrelated cases. Eur J Med Genet 2009;52:291-296.

21. Warburton D. De novo balanced chromosome rearrangements and extra marker chromosomes identified at prenatal diagnosis: clinical significance and distribution of breakpoints. Am J Hum Genet 1991;49:995-1013. 